\section{Allowing gene patents could be an expensive mistake for the US}

Sir-In the discussion of gene patents ${ }^{1}$, it is generally overlooked that these primarily aim at the encoded protein and not the gene. The discoverers try to patent the use of the 'gene product' either as a therapeutic protein, or as an intervention point ('drug target') to treat a disease.

However, although the gene sequence provides necessary information on the protein structure, this information is insufficient, because the mRNA and the original gene product, the protein, are modified in an unpredictable way depending on the temporal and spatial context of their biosynthesis. Who could have predicted the active form of insulin from its gene? Also, the co-receptor of HIV, CCR5, on which a controversial patent has been issued ${ }^{2}$, appears to be a co-receptor for HIV only if it is modified by attachment of sulphate residues ${ }^{3}$. It is evident that, if a patent covers a 'gene product', it should only give rights on the predicted, unmodified form. Experimental evidence for the existence of the predicted function of the gene product should be provided.

What about patenting gene products as drug targets? The EU patent directive states clearly that the human body and its components in their native environment cannot be covered by a patent. What is a drug target other than a component of the human body in its native environment? This statement can only mean that drug targets are not patentable in Europe, otherwise it would be nothing but a political declamation.

There are other problems with patenting drug targets. Many drugs are still discovered using animal model systems or cellular assays. In the United States, for example, the National Institutes of Health has a number of screening programmes using mice to detect anti-epileptic compounds, and cell cultures to detect cytotoxic compounds, where the molecular target is unknown. If a scientist or a company discovers a drug using such an assay, they would be stupid to try to identify the drug target, because there is some likelihood that the drug target is already covered by a patent. Patenting drug targets is therefore scientifically counterproductive: it enforces a culture of 'not wanting to know'.

Let's assume that drug targets can be patented in the United States but not in Europe. As a consequence drugs could be developed unrestrictedly in Europe but not in the United States. US companies would then be forced to move their drug discovery and development to Europe. Still these drugs could not be used in the United States. As a result patients would have to travel from the United States to Europe to receive their life-saving drug. What a great perspective for Europe's biotechnology

and economy!

\section{Hartmut Michel}

Max Planck Institut of Biophysics,

Heinrich-Hoffmann-Straße 7, 60528

Frankfurt am Main, Germany

1. Schiermeier, Q. Nature 406, 111 (2000).
2. Smaglik, P. Nature 404, 322 (2000).
3. Cormier, E. G. et al. Proc. Natl Acad. Sci. USA 97, 5762-5767
(2000).

\section{No room on the carousel for meeting of like minds}

Sir - The day has finally arrived when I receive more scientific meeting announcements than junk mail. I read all the programmes with interest, but wonder about the rationale for such a deluge, when information flows freely over the Internet. Notices of meetings and workshops, scientific discussions, the contents of forthcoming journal issues and many other matters can all be found on the Web. So why travel to a meeting when, in most cases, the information is not even new?

As I open the envelopes, my interest fades rapidly when I see the same speakers and topics regularly repeated. I am not criticizing scientists who circle the globe sharing their discoveries and knowledge with the rest of us, but to me they are on a scientific carousel. Each colourful horse is ridden by a speaker with a lecture in his or her right hand. As the merry-go-round rotates, the topic and speaker will cross your path no matter where you are standing.

So what can justify the existence of scientific meetings? They ought to allow us to communicate and share new advances; to discuss new applications for a technique; to hear the latest evidence supporting a discovery; to develop new projects, and so on.

But the whole principle of a scientific meeting - innovation - is ignored in most of these scientific events. The number of meetings inversely correlates with the amount of interesting data to be communicated. On the scientific carousel, a meeting is held for commercial reasons and people attend out of opportunism (local rivalries, for example) or to visit fancy places. In meetings held by scientific societies, however, the spirit of science is maintained, as participants share and discuss advances and challenge each other verbally.

An individual can communicate exciting results on the Web, but the work cannot be generally accepted until it has been challenged and recognized by fellow researchers at meetings. The recognition and respect of one's peers is what constitutes scientific kinship and justifies society meetings. As for the scientific carousel — like any other fairground attraction, time and market forces will determine its lifespan.

Eleuterio R. Hernandez

Instituto de Bioquimica (CSIC-UCM), Facultad de Farmacia, Universidad Complutense, 28040-Madrid, Spain

\section{Standardized addresses would make web easier}

Sir - This may be the age of information, but gaining access to it is often difficult. Finding a site on the World-Wide Web can be like searching for a needle in a haystack.

In India, only half of the 40 laboratories in the Council for Scientific and Industrial Research (CSIR), the country's largest research and development organization, have websites of their own. These 20 institutions follow five different ways of naming their sites. If you don't know the address, you can't reach the site by guessing. None of them end with .gov.in. The Central Mechanical Engineering Research Institute, Durgapur, has opted for www.cmeri. com. Some end with .org — which gives the impression that the pages are hosted by a service provider located in the United States, where web addresses do not end in a domain indicative of the country. Most web-based search engines are unable to help, since they only reach some $16 \%$ of the web (see Nature 400, 107-109; 1999).

Adopting uniformity in naming domains could circumvent this problem. Many institutions around the world use their name or acronym as the lowest domain and move up hierarchically. For example, all the institutions in the CSIR could use .csir.dsir.gov.in, since CSIR is part of the Ministry of Science and Technology's Department of Scientific and Industrial Research. The Centre for Cellular and Molecular Biology could be at www.ccmb.csir.dsir.gov.in. Educational institutions should follow the same convention. By understanding the organizational hierarchy of any institution one should be able to guess the web address.

The next improvements could be to the content, organization and facilities provided by the web pages. In the scientific world, concerted efforts are under way to realise the full potential of electronic communication, with e-print archives, online journals and the development of E-Biomed. Web pages for government-funded research and development laboratories need to offer more than just basic facts about the organization. They should show depth of content and should include details of their scientific activities, search facilities and routine updates.

Sasidharan Rajkumar

Nagarjuna Group, Nagarjuna Hills, Hyderabad 500 082, India 